\title{
Interhemispheric auditory connectivity: structure and function related to auditory verbal hallucinations
}

\author{
Saskia Steinmann, Gregor Leicht and Christoph Mulert* \\ Psychiatry Neuroimaging Branch, Department of Psychiatry and Psychotherapy, University Medical Center Hamburg-Eppendorf, Hamburg, Germany
}

\section{Edited by:}

Srikantan S. Nagarajan, University of California, San Francisco, USA

\section{Reviewed by:}

Karuna Subramaniam, University of California, San Francisco, USA

Julia P. Owen, University of

California, San Francisco, USA

Georg Juckel, Ruhr University

Bochum, Germany

*Correspondence:

Christoph Mulert, Klinik für

Psychiatrie und Psychotherapie,

Universitätsklinikum

Hamburg-Eppendorf,

Forschungsbereich Bildgebung

Haus W37, Martinistr. 52, D-20246

Hamburg, Germany

e-mail:c.mulert@uke.de
Auditory verbal hallucinations (AVH) are one of the most common and most distressing symptoms of schizophrenia. Despite fundamental research, the underlying neurocognitive and neurobiological mechanisms are still a matter of debate. Previous studies suggested that "hearing voices" is associated with a number of factors including local deficits in the left auditory cortex and a disturbed connectivity of frontal and temporoparietal language-related areas. In addition, it is hypothesized that the interhemispheric pathways connecting right and left auditory cortices might be involved in the pathogenesis of AVH. Findings based on Diffusion-Tensor-Imaging (DTI) measurements revealed a remarkable interindividual variability in size and shape of the interhemispheric auditory pathways. Interestingly, schizophrenia patients suffering from AVH exhibited increased fractional anisotropy (FA) in the interhemispheric fibers than non-hallucinating patients. Thus, higher FA-values indicate an increased severity of AVH. Moreover, a dichotic listening (DL) task showed that the interindividual variability in the interhemispheric auditory pathways was reflected in the behavioral outcome: stronger pathways supported a better information transfer and consequently improved speech perception. This detection indicates a specific structure-function relationship, which seems to be interindividually variable. This review focuses on recent findings concerning the structure-function relationship of the interhemispheric pathways in controls, hallucinating and non-hallucinating schizophrenia patients and concludes that changes in the structural and functional connectivity of auditory areas are involved in the pathophysiology of $\mathrm{AVH}$.

Keywords: auditory-verbal hallucinations, schizophrenia, structural and functional interhemispheric connectivity, auditory cortex, neuroimaging

\section{INTRODUCTION}

Auditory verbal hallucinations (AVH) are one of the most prominent symptoms in schizophrenia (SZ), affecting approximately $70 \%$ of patients (Schneider, 1957), a fact reflected in their use as a major diagnostic criterion for the disease (Diagnostic and Statistical Manual IV, 1994). AVH are simply defined as vocal perceptions without an appropriate external stimulus (Woodruff, 2004). They mostly occur as voices (i.e., "hearing voices") that might comment on or criticize the patient and are therefore thought to reflect dysfunctional auditory processing in speechrelevant brain areas.

Various theoretical models have been proposed regarding the cognitive mechanisms underlying AVH: one currently influential hypothesis is that $\mathrm{AVH}$ represent internally-generated speech (i.e., thinking in words) misidentified as coming from outside the self because of defective self-monitoring (Frith, 1995; for review see Waters et al. (2012). This is assumed to result from a dysfunction in the "forward-model" system, the role of which consists in predicting the sensory consequences of actions. Consequently, patients are not in control of their own inner speech (Frith, 2005).

Despite a large body of research in this area, the etiology of AVH remains unknown and the underlying physiological causes are still not fully understood. While structural neuroimaging studies of AVH initially focused on gray matter (GM) abnormalities in the left auditory cortex, more recent studies have instead emphasized the role of changes in fronto-temporal white matter (WM) fiber tracts relevant for speech. Currently, AVH is thought to result not only from focal impairments in single speech-related areas, but also from altered functional connectivity between these and other brain regions. Thus, research on the neurobiology of $\mathrm{AVH}$ emphasizes a network view postulating dysfunctional interactions among a range of brain regions and functions.

This review begins with a brief overview of structural and functional neuroimaging findings on AVH. Included are studies published between 1990 and 2013 that have used either electroencephalography (EEG, Berger, 1929) or magnetic resonance imaging (MRI) techniques such as voxel-based morphometry (VBM, Ashburner and Friston, 2000), diffusion-tensor-imaging (DTI, Basser et al., 1994), and functional MRI (fMRI). The main section of the review concentrates on findings regarding interhemispheric connectivity between bilateral auditory cortices.

\section{NEUROIMAGING STUDIES ON AVH: CURRENT STATE OF KNOWLEDGE \\ STRUCTURAL NEUROIMAGING}

Research into the pathophysiology of AVH has boomed in the past two decades thanks to the advent of modern neuroimaging 
methods based on MRI techniques (e.g., VBM), which enabled researchers to gain initial insights into the brain regions and networks involved in this challenging symptom. One of the most extensively investigated brain regions in this regard is the left temporal lobe, in particular the primary (PAC or Heschl's gyrus), and secondary (SAC) auditory cortex. These cortical areas are known to be crucial for auditory perception and comprehension (Friederici, 2011).

Accordingly, in patients who suffer from AVH reduced gray matter volume (GMV) has been reported in predominantly speech-related brain areas such as the left superior temporal gyrus (STG) (Barta et al., 1990; Shenton et al., 1992), Heschl's gyrus (Gaser et al., 2004; Nenadic et al., 2010), left middle temporal gyrus (MTG), and left temporo-parietal regions (for a detailed review see Allen et al.,, 2008). A recent meta-analysis of VBM studies that included a total of 438 patients (307 with AVHs) confirmed a significant correlation between the extent of volume loss in the Heschl's gyrus and severity of AVH (Modinos et al., 2013). Marginal GMV reductions were also noted in the right STG, thus suggesting that both the left and right STG are involved in the structural pathology of AVH.

Further, evidence so far suggests that GMV reductions in areas other than sensory cortices also contribute to hallucinatory experiences. Such areas include the insula bilaterally, left amygdala, left inferior frontal gyrus (IFG), parahippocampal gyrus, anterior and posterior cingulate cortex (ACC and PCC), thalamus, cerebellum, and precuneus (Allen et al.,, 2008; García-Martí et al., 2012). More specifically, the insula and the amygdala-key regions for emotional regulation-are postulated to be involved in the commonly observed emotional load of AVH (Alba-Ferrara et al., 2012), while dysfunction of the PCC-known to constitute a basic component in generating a model of the self-is responsible for defective self-referential processing (Northoff and Bermpohl, 2004).

In brief, changes in the auditory cortex and other languagerelated brain areas are the most consistent and replicated findings in structural imaging studies of AVH. However, other cortical regions and the limbic system must also be taken into account, especially for neuropsychological models.

\section{FUNCTIONAL NEUROIMAGING}

Findings of the aforementioned structural studies are supported by several functional imaging studies demonstrating that "hearing voices" involves similar brain regions as normal speech perception and production. For example, acute experience of AVH has been associated with increased activation in bilateral auditory cortices, including the Heschl's gyrus (Dierks et al., 1999; Lennox et al., 2000; van de Ven et al., 2005) and Broca's area (McGuire et al., 1993). Moreover, Shergill et al. (2000a) associated AVH with activations in the inferior frontal cortex and insula, ACC, MTG bilaterally (though somewhat lateralized to the right), the right thalamus and inferior colliculus, as well as the left hippocampus and parahippocampal cortex. Thus, regions not directly related to language such as the left hippocampus/parahippocampusan area thought to be involved in verbal memory-also exhibit increased activation during AVH. Based on this finding, it has been suggested that aberrant retrieval of verbal memories might be one of the factors associated with the involuntary emergence of AVH (Jardri et al., 2011).

In addition, Woodruff et al. (1997) also reported that hallucinating patients demonstrate greater activity in the right than left SAC while listening to speech, i.e., they exhibit a reversed pattern of the typical (left-lateralized) auditory cortex response; this finding was interpreted as a possible consequence of altered interhemispheric connectivity (Woodruff, 2004). Interestingly, a current meta-analysis of dichotic listening studies has suggested reduced left-hemispheric language lateralization as a strong trait marker for the occurrence of AVH within the SZ population (Ocklenburg et al., 2013).

Further, an fMRI study looking into the time course of brain activation immediately prior to AVH onset described activations in the left anterior insula and right MTG, as well as deactivation in the ACC and parahippocampal gyrus (Hoffman et al., 2008). Notably, two previous studies had also reported increased activation in the right MTG prior to the onset of AVH. It is hypothesized that these neural events trigger hallucinatory experiences or predispose patients to them (Lennox et al., 1999; Shergill et al., 2004).

In another study, fMRI was applied to identify neural activation patterns during tasks tapping into verbal self-monitoring processes in patients predisposed to $\mathrm{AVH}$, while they were imagining external auditory speech; attenuated activation was found in the posterior cerebellum, hippocampi, lenticular nuclei bilaterally, the right thalamus, MTG and STG, and left nucleus accumbens (Shergill et al., 2000b). These structures have been suggested to serve as comparators in models of cognitive self-monitoring (Gray et al., 1991), and are also associated with memory retrieval (Henke et al., 1999). Thus, reduced activation during auditory verbal imagery might be related to defective auditory-verbal recall in patients.

Finally, a coordinate-based meta-analysis of $10 \mathrm{fMRI}$ studies looking into the brain areas that are predominantly activated during the experience of AVH provided support for the aforementioned findings and also identified a more widely distributed fronto-temporal language-related network involving the left MTG and STG, Broca's area, bilateral frontal operculum, bilateral anterior insula, left precentral gyrus, and left supramarginal gyrus (Jardri et al., 2011).

\section{FUNCTIONAL AND STRUCTURAL CONNECTIVITY STUDIES}

As mentioned earlier, one current model of AVH suggests that they arise from a disability of patients to recognize self-generated inner speech (Frith, 1995). This model postulates a disturbance in the mechanisms that serve to predict the outcome of inner speech (forward model). Many models of AVH conceptualize the symptom in terms of a failure to integrate information regarding inner speech, generated in the frontal cortex, with sensory processing in temporal and parietal regions (Friston and Frith, 1995; Frith, 2005). There is increasing experimental support for this hypothesis: for example, reduced fronto-temporal connectivity has been observed in SZ patients with AVH during a sentence-completion task (Lawrie et al., 2002), external speech processing (Mechelli et al., 2007), or speaking aloud (Ford et al., 2002). More recently, a resting-state fMRI study 
demonstrated reduced functional connectivity between the left temporo-parietal junction-a critical node for speech perception and AVH genesis (Hoffman et al., 2003) —and the right frontal homotope of Broca's area-associated with speech production in hallucinating patients (Vercammen et al., 2010). In addition, more severe AVH were associated with reduced neural synchrony between the left temporo-parietal junction and bilateral ACC as well as bilateral amygdala; again, these results suggest a dysfunctional interaction among brain areas involved in self-referential processing, attribution of agency, and attentional control (Vercammen et al., 2010).

The introduction of Diffusion Tensor Imaging (DTI) has made examination of specific WM fiber tract characteristics (size, shape, spatial organization) possible (Basser et al., 1994). DTI methods exploit properties of molecular diffusion and characterization of the diffusion tensor to infer the orientation and integrity of WM tracts in the brain. Diffusion is subjected to certain restrictions such as the type of cellular tissue, or the presence of myelin sheaths or cell membranes. As a result, the spatial orientation of fibers can be inferred from its main direction (Mori et al., 1999; Nucifora et al., 2007). The most commonly used metric of diffusion anisotropy is "fractional anisotropy" (FA), a value which varies on a scale from 0 (isotropic) to 1 (anisotropic) (Pierpaoli and Basser, 1996), whereby higher values indicate more parallel alignment of cell membranes or other bio-barriers. In clinical studies, FA values are routinely used as an index of WM integrity and are sensitive to WM deterioration in neurodegenerative and psychiatric disorders (Zhang et al., 2007; Mulert et al., 2011).

In the context of AVH, an early DTI study reported increased FA values of the arcuate fasciculus in hallucinating patients compared to non-hallucinating patients and healthy controls (HC). Since the arcuate fasciculus constitutes the major fiber tract connecting inferior frontal and temporo-parietal areas involved in language perception and production, the above finding indicates stronger connectivity between frontal speech production (i.e., Broca's area) and temporal speech perception areas (i.e., Wernicke's area) in hallucinating patients (Hubl et al., 2004). Interestingly, increased FA values of the arcuate fasciculus have been also demonstrated in non-psychotic subjects suffering from AVH (de Weijer et al., 2011). This implies that alterations of the arcuate fasciculus might be specifically associated with the symptom of AVH rather than SZ.

Another DTI study investigated the arcuate fasciculus in more detail by dividing it into three segments: anterior indirect, posterior indirect and long direct segment (Catani et al., 2011). The anterior indirect segment connects fronto-parietal regions (i.e., Broca's area with Geschwind's area) and appears to relate to speech articulation, whereas the posterior indirect segment connects temporo-parietal regions (i.e., Wernicke's area with Geschwind's area) and is related to speech comprehension (Duffau, 2008). The long direct segment represents the classical arcuate fasciculus. Results showed bilaterally significant reduced FA values in patients, which was however limited to the long direct and the posterior indirect segment, projecting both to the posterior temporal auditory regions associated with speech perception. Interestingly, FA values were lowest in patients with AVH.
However, one earlier study also reported increased FA values in the lateral part of the superior longitudinal fasciculi (SLF) and the ACC in hallucinating patients compared to non-hallucinating patients, while in comparison to healthy controls the FA values were still lower even in the patients who were most prone to AVH (Shergill et al., 2007). The authors suggested that the severity of these changes may vary with the characteristics of symptoms. Further, Makris et al. (2010) demonstrated a significant positive correlation between WM volume in anterior callosal, cingulate and temporal deep WM regions on one hand and positive symptoms, such as AVH or delusions, on the other.

\section{INTERHEMISPHERIC AUDITORY CONNECTIVITY IN HEALTHY CONTROLS (HC)}

While all of the factors mentioned above may be involved in the genesis of $\mathrm{AVH}$, the following part will concentrate on the role of interhemispheric pathways between right and left auditory cortices (see Figure 1).

\section{INTERHEMISPHERIC AUDITORY PATHWAY STRUCTURE}

Interhemispheric interaction is primarily subserved by the brain's commissural system, including the corpus callosum (CC), anterior commissure, posterior commissure, cerebellar commissures, and interthalamic adhesions (Hoptman and Davidson, 1994). The CC is by far the largest WM structure in the human brain, containing more than 300 million transcallosal fibers (Hofer and Frahm, 2006), which are spatially ordered according to their point of origin in the cortex (Schmahmann and Pandya, 2006). So, for example, fibers connecting frontal areas progress through the most anterior part of the CC (genu), while bilateral temporal, parietal or occipital cortices are connected through the posterior parts (isthmus, splenium) of the CC. Thus, interhemispheric interaction between PAC and SAC-both located at the STG-is primarily mediated by interhemispheric auditory pathways running through the posterior third of the CC (isthmus, splenium) (Aboitiz et al., 1992; Bamiou et al., 2007). Moreover, the diameter of various CC subregions varies widely, ranging from small $(0.4-1 \mu \mathrm{m})$ to large $(>3 \mu \mathrm{m})$. Interestingly, interhemispheric

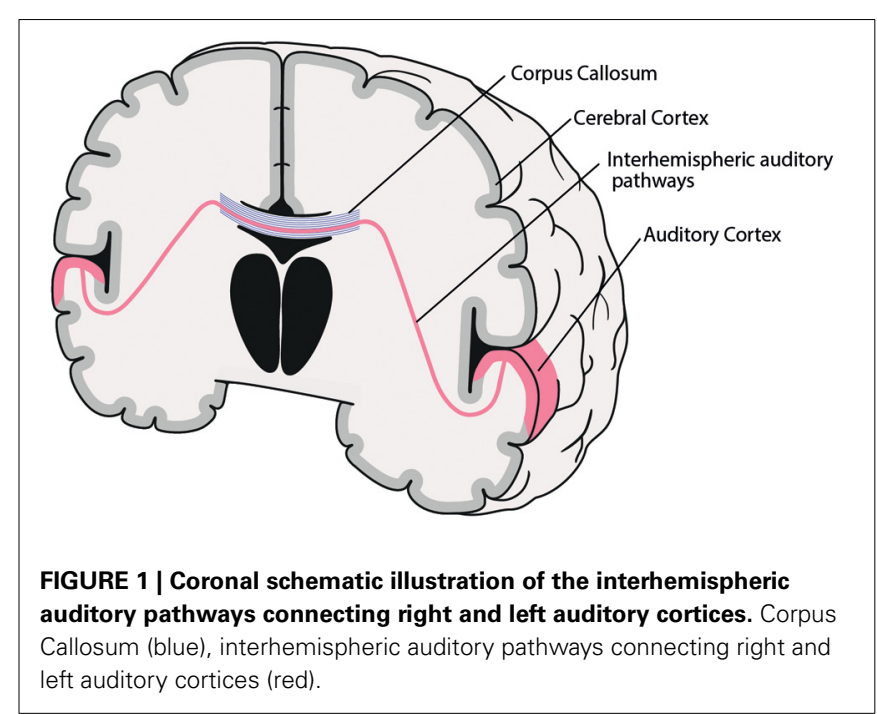


auditory pathways are among the densest and present the largest fiber diameters $(>3 \mu \mathrm{m})$, and thus possess fast conduction velocities, which are assumed to be necessary for fast bilateral interaction, e.g., for sound localization in space (Aboitiz et al., 1992).

\section{INTERHEMISPHERIC AUDITORY PATHWAY FUNCTION}

The functional relevance of interhemispheric auditory fibers was investigated in an event-related potential study that examined interhemispheric interaction of prosodic and syntactic information during speech comprehension in patients with lesions of the posterior third of the CC (Friederici et al., 2007). Results demonstrated that the posterior third of the CC-where interhemispheric auditory fibers cross-is crucial for the integration of prosodic information (right hemisphere) with syntactic information (left hemisphere), which is in turn a prerequisite for spoken language comprehension. In the study by Friederici et al., participants performed a comprehension task that required successful integration of prosodic and syntactic information. Patients with lesions of the posterior part of the CC exhibited an absence of the N400, an event-related potential assumed, in this context, to reflect successful interaction between right and left auditory areas. Thus, failure to produce the N400 potential in patients was interpreted by the authors as evidence for a disconnection of interhemispheric auditory pathways that leads to disrupted information flow among regions associated with lateralized language functions.

Similar results were obtained in studies using the dichotic listening (DL) task. In this paradigm, two different consonantvocal (CV)-syllables such as "ba" or "ga" are paired and presented simultaneously, one in the right ear (RE) and another one in the left ear (LE). A typical finding across studies in healthy righthanded individuals is the so-called Right Ear Advantage (REA), i.e., subjects report the syllable presented to the RE with better accuracy than the one presented to the LE (Kimura, 1961; Hugdahl, 2003). The REA reflects the dominance of the left hemisphere in speech perception as well as the fact that, under DL conditions, auditory information is transferred predominantly through contralateral auditory pathways, while ipsilateral pathways are inhibited (Brancucci et al., 2004). According to the "callosal relay model," the CV-syllable arriving from the LE to the non-dominant right hemisphere requires additional interhemispheric transfer across the CC before it can be efficiently processed in the dominant left hemisphere (Kimura, 1967; Bryden, 1988; Hugdahl, 1995). In this regard, the LE-report serves as an index of interhemispheric transfer.

Pollmann et al. (2002) reported that patients with lesions in the posterior part of the CC did not report any syllables presented to the LE, showing an almost perfect REA. Moreover, studies in patients with multiple sclerosis have revealed a reduced LE report, which correlated with the extent of atrophy of the posterior part of the CC (Barkhof et al., 1998; Pelletier et al., 2001; Gadea et al., 2002). These results indicate that the perception of CV-syllables presented to the LE depends on the integrity of interhemispheric auditory pathways.

In summary, interhemispheric auditory pathways appear to play a crucial role in healthy auditory processing and speech comprehension, such that impaired interhemispheric connectivity leads to disrupted information flow between homologous auditory areas and, consequently, to disturbance of language-related processes.

\section{STRUCTURE-BEHAVIOR RELATIONSHIP OF THE INTERHEMISPHERIC AUDITORY PATHWAYS}

Recent DTI and tractography studies have revealed striking interindividual variability in the size and shape of interhemispheric auditory pathways in the general population (Hofer and Frahm, 2006; Dougherty et al., 2007; Westerhausen et al., 2009). For instance, Westerhausen et al. (2009) investigated the functional relevance of callosal fiber tract interindividual variability in $17 \mathrm{HC}$ with probabilistic DTI tractography. The authors identified the callosal substrate connecting the posterior STG with the left and right Heschl's gyrus and subsequently correlated structural data with individual performance on a DL task of CV-syllables. Results showed that interindividual variation of callosal topography was reflected in behavioral performance: individuals with stronger anatomical pathways between bilateral auditory cortices exhibited better information processing and, consequently, improved perception of the CV-syllable presented to the LE.

Contrary findings were published by Dougherty et al. (2007), who investigated interhemispheric callosal fibers in 55 children with a wide range of reading ability using DTI tractography. Their results showed that phonological awareness (a key factor in reading acquisition) was positively correlated with radial diffusivity of the interhemispheric pathways connecting temporal lobes. Based on these findings, the authors argued that good readers presumably have a higher proportion of large axons but also fewer callosal connections in total and, hence, reduced interhemispheric connectivity.

To the best of our knowledge, no study has so far concurrently investigated the structural integrity of interhemispheric auditory pathways along with the functional activity of the regions they connect. Nevertheless, the above findings indicate a large interindividual variability regarding the relationship between interhemispheric fiber structure and auditory task performance. In addition, several studies have suggested that dysfunctional connectivity between the left and right auditory cortex may lead to deficits in auditory processing (Barkhof et al., 1998; McKay et al., 2000; Pelletier et al., 2001; Gadea et al., 2002; Pollmann et al., 2002). Interestingly, it has been suggested that alterations in the interhemispheric connectivity attended by a disturbed interaction may be associated with unusual auditory perceptions and/ or $\mathrm{AVH}$ in predisposed individuals (Rossell et al., 2001; Woodruff, 2004; Rossell and Boundy, 2005).

\section{ALTERED STRUCTURAL AND FUNCTIONAL INTERHEMISPHERIC AUDITORY CONNECTIVITY AND AVH}

With use of novel neuroimaging measures of WM structure, altered interhemispheric connectivity between the right and left auditory cortex has been observed in hallucinating patients (see Table 1).

\section{FINDINGS OF INCREASED INTERHEMISPHERIC CONNECTIVITY}

In addition to the aforementioned finding of increased FA values in the arcuate fasciculus in patients with schizophrenia, Hubl 


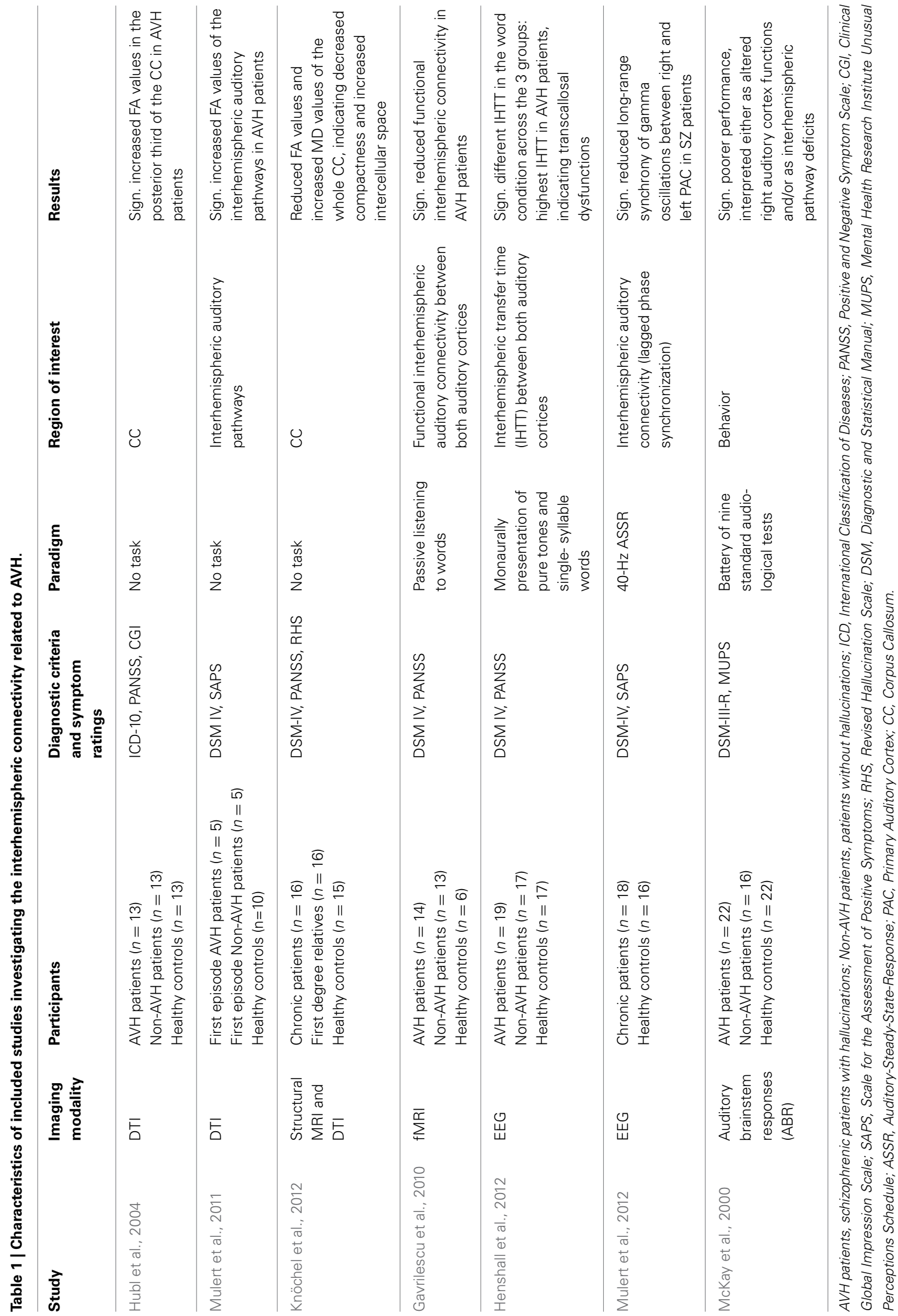


et al. (2004) also reported increased FA values in the posterior third of the CC in 13 acutely ill patients with SZ and frequent AVH compared to 13 acutely patients who had never experienced any hallucinations in their life and HC. When considered as one single group, patients with SZ did not differ from HC regarding FA values. Similar results were reported by a DTI tractography study investigating interhemispheric auditory pathways in relation to $\mathrm{AVH}$ vulnerability in 10 patients with first-episode SZ and $10 \mathrm{HC}$ (Mulert et al., 2011). Again, results indicated no differences between SZ patients and HC. However, when the SZ group was split into two subgroups according to the presence or absence of AVH (each 5 patients), significantly increased FA values were found in hallucinating patients in comparison to non-AVH patients and $\mathrm{HC}$.

Both of the above studies suggest that enhanced transcallosal interhemispheric interaction is associated with increased severity of AVH. This conclusion is consistent with findings of neuropsychological studies that have demonstrated reduced REA or even a left ear advantage (LEA) in the DL task in patients with SZ, especially those suffering from AVH (Green et al., 1994; Bruder et al., 1995; Rossell and Boundy, 2005; Hugdahl et al., 2008): Green et al. (2008) reported complete absence of a REA in hallucinating patients, indicating an almost perfect LEA. Moreover, Hugdahl et al. (2008) found a negative correlation between the ability to report the RE stimulus and BPRS (Brief Psychiatric Rating Scale) hallucination scores in 87 right-handed SZ patients, using a DL-task of CV-syllables.

Hugdahl et al. interpreted their finding of reduced REA as reflecting disturbed left temporal lobe function. However, in light of connectivity studies presented above, an alternative explanation for the above findings might be a reduced hemispheric asymmetry in patients, possibly resulting from increased interhemispheric information transfer between the right and left auditory cortex: taking into account the suggestion by Westerhausen et al. (2009) that stronger anatomical interhemispheric pathways between auditory cortices lead to improved LE-report, as well as findings by Hubl et al. (2004) and Mulert et al. (2011) indicating stronger interhemispheric connectivity in hallucinating patients, one might expect an increased LE-report in patients suffering from AVH.

\section{FINDINGS OF DECREASED INTERHEMISPHERIC CONNECTIVITY}

The above findings of increased interhemispheric connectivity in patients with SZ have not always been replicated: opposite results were reported by Gavrilescu et al. (2010), who used fMRI to examine interhemispheric connectivity between bilateral PAC and SAC cortices in 14 SZ patients currently experiencing $\mathrm{AVH}, 13$ patients without any lifetime history of AVH, and 16 HC. Hallucinating patients exhibited significantly reduced interhemispheric connectivity between auditory cortices compared to patients without $\mathrm{AVH}$ and $\mathrm{HC}$, conceivably reflecting structural disconnection. No significant differences were found between patients without any history of AVH and HC.

A recent DTI study also reported reduced CC volume in 16 chronic SZ patients and their first-degree relatives, which was more pronounced in the posterior genu, isthmus, and splenium (Knöchel et al., 2012). Moreover, decreased FA values in the isthmus and increased mean diffusivity (MD) values of the whole CC and isthmus were observed in patients and their unaffected relatives, indicating decreased fiber density and increased intercellular space. Notably, severity of AVH was associated with volumetric decrease of the CC and reduced fiber integrity.

Thus, both of the above studies suggest reduced interhemispheric connectivity as the basis of $\mathrm{AVH}$, quite in contrast to aforementioned findings of enhanced interhemispheric auditory pathways in hallucinating patients. Non-neuroimaging support for reduced interhemispheric connectivity is supplied by a study by McKay et al. (2000), who employed a battery of nine standard audiological tests to study central auditory processing in 22 currently hallucinating patients with SZ, 16 non-hallucinating patients, and $22 \mathrm{HC}$. Hallucinating patients exhibited significantly poorer performance in a frequency tone pattern test and a staggered spondaic words test compared to both patients without $\mathrm{AVH}$ and $\mathrm{HC}$, suggesting either a functional disturbance in the right auditory cortex and/or interhemispheric communication deficits.

An EEG study also examined interhemispheric functional connectivity between bilateral auditory cortices in SZ patients with and without $\mathrm{AVH}$ and $\mathrm{HC}$; the measure of interest was interhemispheric transfer time (IHTT) during monaural presentation of pure tones or single-syllable words (Henshall et al., 2012). IHTT was calculated based on temporal information obtained from the latency of the auditory $\mathrm{N} 1$ evoked potential. No differences were found for pure tones, but IHTT differed significantly in the word condition across the 3 groups: Hallucinating patients exhibited the highest IHTT values, whereas in HC IHTT was close to zero, and in non-AVH patients it had a negative value. The authors argued that different IHTT might arise from either transcallosal dysfunction in hallucinating patients or from abnormal cerebral lateralization in non-AVH patients.

Additional evidence for disturbed interhemispheric connectivity in SZ has been provided by an EEG study that investigated the functional connectivity between right and left primary and secondary auditory cortices in 18 chronic SZ patients and 16 $\mathrm{HC}$, using phase synchronization in the context of a $40 \mathrm{~Hz}$ auditory steady-state response task (ASSR) (Mulert et al., 2012). The major finding was reduced long-range synchrony of gamma oscillations between the right and left primary auditory cortex (PAC) in patients, but not between bilateral secondary auditory cortices. Notably, reduced interhemispheric gamma synchrony was associated with auditory hallucination scores, suggesting that disturbed interhemispheric connectivity between bilateral PAC in the gamma band might be relevant for the emergence of AVH.

\section{MIXED FINDINGS AND THE NEED OF FURTHER RESEARCH}

Modern neuroimaging techniques have provided us with initial insights into what is happening in the brain of hallucinating patients. Volumetric and functional studies consistently report structural and functional disturbances predominantly in speech-related brain areas, but also in limbic and non-sensory regions. The existing literature does not support the hypothesis of increased interhemispheric connectivity in patients with $\mathrm{SZ}$ in general, i.e., when the present or absence of AVH is not taken into account. SZ is generally regarded as a disorder of 
dysfunctional connectivity and disturbed integration among neuronal systems, reflected in volume reductions affecting both gray matter and WM regions (Friston and Frith, 1995; Csernansky and Cronenwett, 2008). In hallucinating patients however, more recent studies using DTI have revealed both increased and decreased interhemispheric connectivity between the right and left auditory cortex (Hubl et al., 2004; Mulert et al., 2011; Knöchel et al., 2012).

On the one hand, findings of more prominent interhemispheric pathways (Hubl et al., 2004; Mulert et al., 2011) support the theory of "hyperconnectivity" in hallucinating patients, as suggested by John et al. (2008). These authors used MRI to investigate the whole CC and its subregions and observed in SZ patients a significant volume increase in the anterior truncus, which possibly comprises interhemispheric fibers that connect temporal association cortices bilaterally. Accordingly, they suggested that an "abnormal functional hyperconnection" might be involved in the emergence of positive SZ symptoms.

According to the above it might be postulated that, although increased interhemispheric auditory connectivity in healthy participants appears to improve speech perception, it might in contrast be disadvantageous for SZ patients, leading to increased severity of "hearing voices." Thus, one assumption is that stronger interhemispheric connectivity-possibly in combination with further disturbances in the STG or in fronto-temporal connections-might contribute to the emergence of AVH in predisposed individuals (Mulert et al., 2011).

This idea fits nicely to a recent MRI study examining the midsagittal cross-sectional area of the CC with regard to tinnitus (Diesch et al., 2012), which is-similar to AVH-an auditory phantom percept that is experienced in the absence of an external auditory stimulus. Interestingly, also tinnitus patients demonstrated larger volume of the splenium (the CC area where the interhemispheric auditory pathways cross). Moreover, subjective intrusiveness of tinnitus was correlated positively with the size of the majority of the CC subregions, including the posterior parts. Assuming that it is hypersynchronous firing of neurons in the auditory cortex that represents the neurophysiological correlate of tinnitus (Eggermont, 2007), Diesch and colleagues suggested that stronger interhemispheric auditory pathways may facilitate the development and persistence of a positive feedback loop between tinnitus generators located in both hemispheres. Thus, it could be hypothesized that stronger interhemispheric connectivity between auditory areas represent one part of the underlying pathophysiology involved in auditory phantom precepts such as AVH or tinnitus.

On the other hand, one DTI study (Knöchel et al., 2012) and one fMRI-study (Gavrilescu et al., 2010) have demonstrated reduced interhemispheric connectivity in patients, supporting the "disconnectivity hypothesis" of SZ (Friston and Frith, 1995). Moreover, EEG findings of longer IHTT (Henshall et al., 2012) or decreased gamma synchrony (Mulert et al., 2012) indicate reduced interhemispheric connectivity in hallucinating patients. Gavrilescu et al. (2010) have suggested that such disrupted integration of information between the right and left auditory cortex gives rise to aberrant auditory processing, which might be experienced as "external voices." Since primary, secondary, and auditory association cortices are specialized for different integrative speech functions, it is conceivable that the above disturbances might lead to extensive deficits ranging from basic auditory processing to higher-order language processing.

Altogether, at present there is insufficient neuroimaging evidence to fully depict the structural and functional contribution of interhemispheric pathways to $\mathrm{AVH}$, because of the wide discrepancy of findings. Current evidence suggests some involvement of interhemispheric pathways in the pathophysiology of $\mathrm{AVH}$, but their specific role remains ambiguous. However, reviewed studies all have one finding in common: significant differences concerning FA values of interhemispheric pathways are only found when comparing hallucinating patients to patients with no lifetime experience of AVH or HC. Patients without AVH do not differ from HC, suggesting that interhemispheric connectivity disturbances are specific to the presence of AVH.

The observed inconsistencies among studies might be due to methodological differences. Therefore, and because of the small number of existing studies, it is difficult to draw generalizable conclusions, especially when subgroups (e.g., AVH vs. non-AVH patients) are investigated. More specifically, phenomenological features of hallucinating experiences (such as loudness, perceived source-external vs. internal-, number and/or overlap of voices, type, etc.) are not reported in detail, such that their effect on findings cannot be considered. Most commonly, neuroimaging research has so far tended to neglect phenomenological features, making only a crude distinction between hallucinating and non-hallucinating patients. Similarly, other important variables such as age, duration of illness, medication, and clinical status (first-episode, acute exacerbation, chronicity) might affect results and need to be considered in future studies. A clearly defined clinical diagnosis and a careful phenomenological assessment are essential in order to elucidate the association between specific hallucinatory characteristics and functional/structural deficits and also to enhance comparability of findings. Thus, one next step to progress in the understanding of AVH might be a longitudinal approach to study developmental trends toward an increase or decrease of the interhemispheric fibers' integrity across the clinical status (prodromal, first-episode, and chronicity patients with $\mathrm{AVH}$, ideally matched with non-hallucinating patients).

One potential approach for the investigation of functional connectivity between both auditory cortices might be restingstate (rs) fMRI using independent component analysis (ICA). This approach has been used several times (Mantini et al., 2007; Doucet et al., 2011; Arbabshirani et al., 2013; Storti et al., 2013) identifying commonly a bilateral auditory component covering the primary and secondary auditory areas within Heschl's gyrus and STG. Although there have been ICA/rs-fMRI studies focused on SZ (Mannell et al., 2010; Yu et al., 2013), the topic of AVH has not yet been addressed. However, in the broader context of auditory phantom precepts, there have been studies of tinnitus (Kim et al., 2012; Maudoux et al., 2012; Davies et al., 2013, for review see Husain and Schmidt, 2014) that demonstratedamong others-altered functional interhemispheric connectivity between bilateral auditory cortices. Thus, rs-fMRI could be useful to investigate possible alterations in resting-state neuronal activity 
between auditory and non-auditory networks in hallucinating patients.

So far, there have been no multimodal studies that have conjointly investigated structural aspects of interhemispheric pathways and functional activations of the regions they connect. Such a framework-e.g., by combining simultaneous EEG-fMRI (with high temporal and spatial resolution) and DTI-that interfaces functional and structural neuroimaging methods would facilitate progress in understanding the precise contribution of the interhemispheric auditory connectivity to AVH and could provide new insights into the involved brain networks. Further, this approach would help clarify whether interhemispheric fiber tracts are reduced or increased in integrity or amount, or possibly misdirected.

\section{CONCLUSION}

In conclusion, the present review provides an up-to-date summary of findings from studies examining the role of interhemispheric auditory connectivity in the pathophysiology of AVH across different modalities (brain structure, function, and behavior) and populations (HC, SZ patients with and without AVH). The most consistent findings across studies are functional and structural interhemispheric connectivity disturbances in hallucinating patients compared to non-hallucinating patients and $\mathrm{HC}$. Future research on AVH needs to address methodological limitations of existing studies such as the failure to take into account phenomenological features and clinical status. Moreover, studies investigating both structural and functional aspects of interhemispheric connectivity and their relationship are warranted to improve our understanding of the precise contribution of interhemispheric fibers to $\mathrm{AVH}$ and to gain new insights into the neurophysiological mechanisms underlying AVH.

\section{ACKNOWLEDGMENTS}

We wish to thank Shelley Esposito for helpful language editing. This research was supported by the SFB 936 C6 (to Christoph Mulert and Gregor Leicht) of the German Research Foundation. Saskia Steinmann was supported by a grant from the Werner Otto Foundation, Hamburg, Germany. This work is part of Saskia Steinmann's doctoral thesis (Dr. rer. nat.).

\section{REFERENCES}

Aboitiz, F. (1992). Brain connections: interhemispheric fiber systems and anatomical brain asymmetries in humans. Biol. Res. 25, 51-61.

Aboitiz, F., Scheibel, A. B., Fisher, R. S., and Zaidel, E. (1992). Fiber composition of the human corpus callosum. Brain Res. 598, 143-153. doi: 10.1016/00068993(92)90178-C

Alba-Ferrara, L., Fernyhough, C., Weis, S., Mitchell, R. L., and Hausmann, M. (2012). Contributions of emotional prosody comprehension deficits to the formation of auditory verbal hallucinations in schizophrenia. Clin. Psychol. Rev. 32, 244-250. doi: 10.1016/j.cpr.2012.02.003

Allen, P., Laroi, F., McGuire, P. K., and Aleman, A. (2008). The hallucinating brain: a review of structural and functional neuroimaging studies of hallucinations. Neurosci. Biobehav. Rev. 32, 175-191. doi: 10.1016/j.neubiorev.2007.07.012

Arbabshirani, M. R., Havlicek, M., Kiehl, K. A., Pearlson, G. D., and Calhoun, V. D. (2013). Functional network connectivity during rest and task conditions: a comparative study. Hum. Brain Mapp. 34, 2959-2971. doi: 10.1002/ hbm. 22118

Ashburner, J., and Friston, K. J. (2000). Voxel-based morphometry-the methods. Neuroimage. 11(6 Pt 1), 805-821. doi: 10.1006/nimg.2000.0582
Bamiou, D. E., Sisodiya, S., Musiek, F. E., and Luxon, L. N. (2007). The role of interhemispheric pathways in hearing. Brain Res. Rev. 56, 170-182. doi: 10.1016/j.brainresrev.2007.07.003

Basser, P. J., Mattiello, J., and LeBihan, D. (1994). MR diffusion tensor spectroscopy and imaging. Biophys J. 66, 259-267. doi: 10.1016/S0006-3495(94)80775-1

Barkhof, F., Elton, M., Lindeboom, J., Tas, M. W., Schmidt, W. F., Hommes, O. R., et al. (1998). Functional correlates of callosal atrophy in relapsing-remitting multiple sclerosis patients. A preliminary MRI study. J. Neurol. 245, 153-158. doi: 10.1007/s004150050196

Barta, P. E., Pearlson, G. D., Powers, R. E., Richards, S. S., and Tune, L. E. (1990). Auditory hallucinations and smaller superior temporal gyral volume in schizophrenia. Am. J. Psychiatry 147, 1457-1462.

Berger, H. (1929). Über das Elektrenkephalogramm des Menschen. Arch. $f$ Psychiatr. 87, 527-570. doi: 10.1007/BF01797193

Brancucci, A., Babiloni, C., Babiloni, F., Galderisi, S., Mucci, A., Tecchio, F., et al. (2004). Inhibition of auditory cortical responses to ipsilateral stimuli during dichotic listening: evidence from magnetoencephalography. Eur J. Neurosci. 19, 2329-2336. doi: 10.1111/j.0953-816X.2004.03302.x

Bruder, G., Rabinowicz, E., Towey, J., Brown, A., Kaufmann, C. A., Amador, X., et al. (1995). Smaller right ear (left hemisphere) advantage for dichotic fused words in patients with schizophrenia. Am. J. Psychiatry 152, 932-935.

Bryden, M. P. (1988). "An overview of the DL procedure and its relation to cerebral organization," in Handbook of Dichotic Listening: Theory, Methods and Research, ed K. Hugdahl (Chichester: John Wiley \& Sons.), 1-44.

Catani, M., Craig, M. C., Forkel, S. J., Kanaan, R., Picchioni, M., Toulopoulou, T., et al. (2011). Altered integrity of perisylvian language pathways in schizophrenia: relationship to auditory hallucinations. Biol. Psychiatry 70, 1143-1150. doi: 10.1016/j.biopsych.2011.06.013

Csernansky, J. G., and Cronenwett, W. J. (2008). Neural networks in schizophrenia. Am. J. Psychiatry 165, 937-939. doi: 10.1176/appi.ajp.2008.08050700

Davies, J., Gander, P. E., Andrews, M., and Hall, D. A. (2013). Auditory network connectivity in tinnitus patients: a resting-state fMRI study. Int. J. Audiol. doi: 10.3109/14992027.2013.846482. [Epub ahead of print].

de Weijer, A. D., Neggers, S. F., Diederen, K. M., Mandl, R. C., Kahn, R. S., Hulshoff Pol, H. E., et al. (2011). Aberrations in the arcuate fasciculus are associated with auditory verbal hallucinations in psychotic and in non-psychotic individuals. Hum. Brain Mapp. 34, 626-634. doi: 10.1002/hbm.21463

Dierks, T., Linden, D. E., Jandl, M., Formisano, E., Goebel, R., Lanfermann, H., et al. (1999). Activation of Heschl's gyrus during auditory hallucinations. Neuron 22, 615-621. doi: 10.1016/S0896-6273(00)80715-1

Diesch, E., Schummer, V., Kramer, M., and Rupp, A. (2012). Structural changes of the corpus callosum in tinnitus. Front. Syst. Neurosci. 6:17. doi: 10.3389/fnsys.2012.00017

Doucet, G., Naveau, M., Petit, L., Delcroix, N., Zago, L., Crivello, F., et al. (2011). Brain activity at rest: a multiscale hierarchical functional organization. J. Neurophysiol. 105, 2753-2763. doi: 10.1152/jn.00895.2010

Dougherty, R. F., Ben-Shachar, M., Deutsch, D. K., Hernandez, A., Fox, G. R., and Wandell, B. A. (2007). Temporal-callosal pathway diffusivity predicts phonological skills in children. Proc. Natl. Acad. Sci. U.S.A. 104, 8556-8561. doi: 10.1073/pnas.0608961104

Duffau, H. (2008). The anatomo-functional connectivity of language revisited. New insights provided by electrostimulation and tractography. Neuropsychologia 46, 927-934. doi: 10.1016/j.neuropsychologia.2007. 10.025

Eggermont, J. J. (2007). Correlated neural activity as the driving force for functional changes in auditory cortex. Hear. Res. 229, 69-80. doi: 10.1016/j.heares.2007.01.008

Ford, J. M., Mathalon, D. H., Whitfield, S., Faustman, W. O., and Roth, W. T. (2002). Reduced communication between frontal and temporal lobes during talking in schizophrenia. Biol. Psychiatry 51, 485-492. doi: 10.1016/S00063223(01)01335-X

Friederici, A. D. (2011). The brain basis of language processing: from structure to function. Physiol. Rev. 91, 1357-1392. doi: 10.1152/physrev.00006.2011

Friederici, A. D., von Cramon, Y. D., and Kotz, S. A. (2007). Role of the corpus callosum in speech comprehension: interfacing syntax and prosody. Neuron 53 , 135-145. doi: 10.1016/j.neuron.2006.11.020

Friston, K. J., and Frith, C. D. (1995). Schizophrenia: a disconnection syndrome? Clin. Neurosci. 3, 89-97. 
Frith, C. D. (1995). The cognitive abnormalities underlying the symptomatology and the disability of patients with schizophrenia. Int. Clin. Psychopharmacol. 10(Suppl. 3), 87-98. doi: 10.1097/00004850-199509000-00012

Frith, C. D. (2005). The neural basis of hallucinations and delusion. C. R. Biol. 328, 169-175. doi: 10.1016/j.crvi.2004.10.012

Gadea, M., Marti-Bonmati, L., Arana, E., Espert, R., Casanova, V., and Pascual, A. (2002). Dichotic listening and corpus callosum magnetic resonance imaging in relapsing-remitting multiple sclerosis with emphasis on sex differences. Neuropsychology 16, 275-281. doi: 10.1037/0894-4105.16.2.275

García-Martí, G., Aguilar, E. J., Martí-Bonmatí, L., Escartí, M., and Sanjuán, J. (2012). Multimodal morphometry and functional magnetic resonance imaging in schizophrenia and auditory hallucinations. World J. Radiol. 4, 159-166. doi: 10.4329/wjr.v4.i4.159

Gaser, C., Nenadic, I., Volz, H. P., Büchel, C., and Sauer, H. (2004). Neuroanatomy of "hearing voices": a frontotemporal brain structural abnormality associated with auditory hallucinations in schizophrenia. Cereb. Cortex 14, 91-96. doi: 10.1093/cercor/bhg107

Gavrilescu, M., Rossell, S., Stuart, G. W., Shea, T. L., Innes-Brown, H., Henshall, K., et al. (2010). Reduced connectivity of the auditory cortex in patients with auditory hallucinations: a resting state functional magnetic resonance imaging study. Psychol. Med. 40, 1149-1158. doi: 10.1017/S0033291709991632

Gray, J. A., Feldon, J., Rawlins, J. N. P., and Smith, A. D. (1991). The neuropsychology of schizophrenia. Behav. Brain Sci. 14, 1-19. doi: 10.1017/S0140525X00065055

Green, M. F., Hugdahl, K., and Mitchell, S. (1994). Dichotic listening during auditory hallucinations in patients with schizophrenia. Am. J. Psychiatry. 151, $357-362$.

Green, A., Straube, B., and Kircher, T. (2008). "Sprachverständnis—Bildgebung," in Neuropsychologie der Schizophrenie. Symptome, Kognition, Gehirn, eds T. Kircher and S. Gauggel (Heidelberg: Springer Medizin Verlag), 332-346.

Henke, K., Weber, B., Kneifel, S., Wieser, H. G., and Buck, A. (1999). Human hippocampus associates information in memory. Proc. Natl. Acad. Sci. U.S.A. 96, 5884-5889. doi: 10.1073/pnas.96.10.5884

Henshall, K. R., Sergejew, A. A., McKay, C. M., Rance, G., Shea, T. L., Hayden, M. J., et al. (2012). Interhemispheric transfer time in patients with auditory hallucinations: an auditory event-related potential study. Int. J. Psychophysiol. 84, 130-139. doi: 10.1016/j.ijpsycho.2012.01.020

Hofer, S., and Frahm, J. (2006). Topography of the human corpus callosum revisited-Compre-hensive fiber tractography using tensor magnetic resonance imaging. Neuroimage 32, 989-994. doi: 10.1016/j.neuroimage.2006.05.044

Hoffman, R. E., Anderson, A. W., Varanko, M., Gore, J. C., and Hampson, M. (2008). Time course of regional brain activation associated with onset of auditory/verbal hallucinations. Br. J. Psychiatry 193, 424-425. doi: 10.1192/bjp.bp. 107.040501

Hoffman, R. E., Hawkins, K. A., Gueorguieva, R., Boutros, N. N., Rachid, F., Carroll, K., et al. (2003). Transcranial magnetic stimulation of left temporoparietal cortex and medication-resistant auditory hallucination. Arch. Gen. Psychiatry 60, 49-56. doi: 10.1001/archpsyc.60.1.49

Hoptman, M. J., and Davidson, R. J. (1994). How and why do the two cerebral hemispheres interact? Psychol. Bull. 116, 195-219. doi: 10.1037/00332909.116.2.195

Hubl, D., König, T., Strik, W., Federspiel, A., Kreis, R., Bösch, C., et al. (2004). Pathways that make voices. White matter changes in auditory hallucination. Arch. Gen. Psychiatry 61, 658-668. doi: 10.1001/archpsyc.61.7.658

Hugdahl, K. (1995). "Dichotic Listening: Probing temporal lobe functional integrity," in Brain Asymmetry, eds R. J. Davidson and K. Hugdahl (Cambridge, MA: MIR Press), 123-156.

Hugdahl, K. (2003). "Dichotic listening in the study of auditory laterality," in The Asymmetrical Brain, eds K. Hugdahl and R. J. Davidson (Cambridge, MA: MIT Press), 441-475.

Hugdahl, K., Løberg, E. M., Jørgensen, H. A., Lundervold, A., Lund, A., Green, M. F., et al. (2008). Left hemisphere lateralisation of auditory hallucinations in schizophrenia: a dichotic listening study. Cogn. Neuropsychiatry 13, 166-179. doi: $10.1080 / 13546800801906808$

Husain, F. T., and Schmidt, S. A. (2014). Using resting state functional connectivity to unravel networks of tinnitus. Hear. Res. 307, 153-162. doi: 10.1016/j.heares.2013.07.010

Jardri, R., Pouchet, A., Pins, D., and Thomas, P. (2011). Cortical activations during auditory verbal hallucinations in schizophrenia: a coordinate-based metaanalysis. Am. J. Psychiatry 168, 73-81. doi: 10.1176/appi.ajp.2010.09101522
John, J. P., Shakeel, M. K., and Jain, S. (2008). Corpus Callosum area differences and gender dimorphism in neuroleptic-naive, recent-onset schizophrenia and healthy control subject. Schizophr. Res. 103, 11-21. doi: 10.1016/j.schres.2008.04.035

Kim, J. Y., Kim, Y. H., Lee, S., Seo, J. H., Song, H. J., Cho, J. H., et al. (2012). Alteration of functional connectivity in tinnitus brain revealed by resting-state fMRI?: a pilot study. Int. J. Audiol. 51, 413-417. doi: 10.3109/14992027.2011.652677

Kimura, D. (1961). Cerebral dominance and the perception of verbal stimuli. Can. J. Psychol. 15, 166-171. doi: 10.1037/h0083219

Kimura, D. (1967). Functional asymmetry of the brain in dichotic listening. Cortex 3, 163-168. doi: 10.1016/S0010-9452(67)80010-8

Knöchel, C., Oertel-Knöchel, V., Schönmeyer, R., Rotarska-Jagiela, A., van de Ven, V., Prvulovic, D., et al. (2012). Interhemispheric hypoconnectivity in schizophrenia: fiber integrity and volume differences of the corpus callosum in patients and unaffected relatives. Neuroimage 59, 926-934. doi: 10.1016/j.neuroimage.2011.07.088

Lawrie, S. M., Buechel, C., Whalley, H. C., Frith, C. D., Friston, K. J., and Johnstone, E. C. (2002). Reduced frontotemporal functional connectivity in schizophrenia associated with auditory hallucinations. Biol. Psychiatry 51, 1008-1011. doi: 10.1016/S0006-3223(02)01316-1

Lennox, B. R., Bert, S., Park, G., Jones, P. B., and Morris, P. G. (1999). Spatial temporal mapping of neural activity associated with auditory hallucinations. Lancet 353, 644. doi: 10.1016/S0140-6736(98)05923-6

Lennox, B. R., Park, S. B., Medley, I., Morris, P. G., and Jones, P. B. (2000). The functional anatomy of auditory hallucinations in schizophrenia. Psychiatry Res. 100, 13-20. doi: 10.1016/S0925-4927(00)00068-8

Makris, N., Seidman, L. J., Ahern, T., Kennedy, D. N., Caviness, V. S., Tsuang, M. T., et al. (2010). White matter volume abnormalities and associations with symptomatology in schizophrenia. Psychiatry Res. 183, 21-29. doi: 10.1016/j.pscychresns.2010.04.016

Mannell, M. V., Franco, A. R., Calhoun, V. D., Cañive, J. M., Thoma, R. J., and Mayer, A. R. (2010). Resting state and task-induced deactivation: a methodological comparison in patients with schizophrenia and healthy controls. Hum. Brain Mapp. 31, 424-37. doi: 10.1002/hbm.20876

Mantini, D., Perrucci, M. G., Del Gratta, C., Romani, G. L., and Corbetta, M. (2007). Electrophysiological signatures of resting state networks in the human brain. Proc. Natl. Acad. Sci. U.S.A. 104, 13170-13175. doi: 10.1073/pnas.0700668104

Maudoux, A., Lefebvre, P., Cabay, J. E., Demertzi, A., Vanhaudenhuyse, A., Laureys, S., et al. (2012). Connectivity graph analysis of the auditory resting state network in tinnitus. Brain Res. 1485, 10-21. doi: 10.1016/j.brainres.2012.05.006

McGuire, P. K., Shah, G. M., and Murray, R. M. (1993). Increased blood flow in Broca's area during auditory hallucinations in schizophrenia. Lancet 342, $703-$ 106. doi: 10.1016/0140-6736(93)91707-S

McKay, C. M., Headlam, D. M., and Copolov, D. L. (2000). Central auditory processing in patients with auditory hallucinations. Am. J. Psychiatry 157, 759-766. doi: 10.1176/appi.ajp.157.5.759

Mechelli, A., Allen, P., Amaro, E. Jr., Fu, C. H., Williams, S. C., Brammer, M. J., et al. (2007). Misattribution of speech and impaired connectivity in patients with auditory verbal hallucinations. Hum. Brain Mapp. 28, 1213-1222. doi: $10.1002 / \mathrm{hbm} .20341$

Modinos, G., Costafreda, S. G., van Tol, M. J., McGuire, P. K., Aleman, A., and Allen, P. (2013). Neuroanatomy of auditory verbal hallucinations in schizophrenia: a quantitative meta-analysis of voxel-based morphometry studies. Cortex 49, 1046-1055. doi: 10.1016/j.cortex.2012.01.009

Mori, S., Crain, B. J., Chacko, V. P., and van Zijl, P. C. M. (1999). Three-dimentional tracking of axonal projections in the brain by magnetic resonance imaging. Ann. Neurol. 45, 265-269.

Mulert, C., Kirsch, V., Pascual-Marqui, R., McCarley, R. W., and Spencer, K. M. (2012). Long-range synchrony of gamma oscillations and auditory hallucination symptoms in schizophrenia. Int. J. Psychophysiol. 79, 55-63. doi: 10.1016/j.ijpsycho.2010.08.004

Mulert, C., Kirsch, V., Whiteford, T., Salisbury, D., Alvarado, J., Pelevin, P., et al. (2011). Hearing voices: the role of interhemispheric auditory connectivity. World J. Biol. Psychiatry 13, 153-158. doi: 10.3109/15622975.2011. 570789

Nenadic, I., Smesny, S., Schlösser, R. G., Sauer, H., and Gaser, C. (2010). Auditory hallucinations and brain structure in schizophrenia: voxel-based morphometric study. Br. J. Psychiatry 196, 412-413. doi: 10.1192/bjp.bp.109.070441 
Northoff, G., and Bermpohl, F. (2004). Cortical midline structures and the self. Trends Cogn. Sci. 8, 102-107. doi: 10.1016/j.tics.2004.01.004

Nucifora, P. G., Verma, R., Lee, S. K., and Melhem, E. R. (2007). Diffusiontensor MR imaging and tractography: exploring brain microstructure and connectivity. Radiology 245, 367-384.

Ocklenburg, S., Westerhausen, R., Hirnstein, M., and Hugdahl, K. (2013). Auditory hallucinations and reduced language lateralization in schizophrenia: a metaanalysis of dichotic listening studies. J. Int. Neuropsychol. Soc. 19, 410-418. doi: 10.1017/S1355617712001476

Pelletier, J., Suchet, L., Witjas, T., Habib, M., Guttmann, C. R., Salamon, G., et al. (2001). A longitudinal study of callosal atrophy and interhemispheric dysfunction in relapsing-remitting multiple sclerosis. Arch. Neurol. 58, 105-11. doi: 10.1001/archneur.58.1.105

Pierpaoli, C., and Basser, P. J. (1996). Toward a quantitative assessment of diffusion anisotropy. Magn. Reson. Med. 36, 893-906. doi: 10.1002/mrm. 1910360612

Pollmann, S., Maertens, M., von Cramon, D. Y., Lepsien, J., and Hugdahl, K. (2002). Dichotic listening in patients with splenial and nonsplenial callosal lesions. Neuropsychol. 16, 56-64.

Rossell, S. L., and Boundy, C. L. (2005). Are auditory-verbal hallucinations associated with auditory affective processing deficits? Schizophr. Res. 78, 95-106. doi: 10.1016/j.schres.2005.06.002

Rossell, S. L., Shapleske, J., Fukuda, R., Woodruff, P. W., Simmons, A., and David, A. S. (2001). Corpus callosum area and functioning in schizophrenic patients with auditory-verbal hallucinations. Schizophr Res. 50, 9-17.

Schmahmann, J. D., and Pandya, D. N. (2006). Fiber Pathways of the Brain. New York, NY: Oxford University Press. doi: 10.1093/acprof:oso/9780195104233. 001.0001

Schneider, K. (1957). Primary \& secondary symptoms in schizophrenia. Fortschr. Neurol. Psychiatry 25, 487-490.

Shenton, M. E., Kikinis, R., Jolesz, F. A., Pollak, S. D., LeMay, M., Wible, C. G., et al. (1992). Abnormalities of the left temporal lobe and thought disorder in schizophrenia. A quantitative magnetic resonance imaging study. N. Eng. J. Med. 327, 604-612. doi: 10.1056/NEJM199208273270905

Shergill, S. S., Brammer, M. J., Williams, S. C., Murray, R. M., and McGuire, P. K. (2000a). Mapping auditory hallucinations in schizophrenia using functional magnetic resonance imaging. Arch. Gen. Psychiatry 57, 1033-1038. doi: 10.1001/ archpsyc.57.11.1033

Shergill, S. S., Bullmore, E., Simmons, A., Murray, R., and McGuire, P. (2000b). Functional anatomy of auditory verbal imagery in schizophrenic patients with auditory hallucinations. Am. J. Psychiatry 157, 1691-1693. doi: 10.1176/appi. ajp.157.10.1691

Shergill, S. S., Brammer, M. J., Amaro, E., Williams, S. C. R., Murray, R. M., and McGuire, P. K. (2004). Temporal course of auditory hallucinations. Br. J. Psychiatry 185, 516-517. doi: 10.1192/bjp.185.6.516

Shergill, S. S., Kanaan, R. A., Chitnis, X. A., O’Daly, O., Jones, D. K., Frangou, S., et al. (2007). A diffusion tensor imaging study of fasciculi in schizophrenia. Am. J. Psychiatry 164, 467-473. doi: 10.1176/appi.ajp.164.3.467
Storti, S. F., Formaggio, E., Nordio, R., Manganotti, P., Fiaschi, A., Bertoldo, A., et al. (2013). Automatic selection of resting-state networks with functional magnetic resonance imaging. Front. Neurosci. 7:72. doi: 10.3389/fnins.2013.00072

van de Ven, V. G., Formisano, E., Röder, C. H., Prvulovic, D., Bittner, R. A., Dietz, M. G., et al. (2005). The spatiotemporal pattern of auditory cortical responses during verbal hallucinations. Neuroimage 27, 644-655. doi: 10.1016/j.neuroimage.2005.04.041

Vercammen, A., Knegtering, H., den Boer, J. A., Liemburg, E. J., and Aleman, A. (2010). Auditory hallucinations in schizophrenia are associated with reduced functional connectivity of the temporo-parietal area. Biol. Psychiatry 67, 912-918. doi: 10.1016/j.biopsych.2009.11.017

Waters, F., Allen, P., Aleman, A., Fernyhough, C., Woodward, T. S., Badcock, J. C., et al. (2012). Auditory hallucinations in schizophrenia and nonschizophrenia populations: a review and integrated model of cognitive mechanisms. Schizophr. Bull. 38, 683-693. doi: 10.1093/schbul/sbs045

Westerhausen, R., Gruner, R., Sprecht, K., and Hugdahl, K. (2009). Functional relevance of interindividual differences in temporal lobe callosal pathways: a DTI tractography study. Cereb. Cortex 19, 1322-1329. doi: 10.1093/cercor/bhn173

Woodruff, P. W. (2004). Auditory hallucinations: insights and questions from neuroimaging. Cogn. Neuropsychiatry 9, 73-91. doi: 10.1080/13546800344000165

Woodruff, P. W., Wright, I. C., Bullmore, E. T., Brammer, M., Howard, R. J., Williams, S. C., et al. (1997). Auditory hallucinations and the temporal cortical response to speech in schizophrenia: a functional magnetic resonance imaging study. Am. J. Psychiatry 154, 1676-1682.

Yu, Q., Sui, J., Kiehl, K. A., Pearlson, G., and Calhoun, V. D. (2013). Staterelated functional integration and functional segregation brain networks in schizophrenia. Schizophr. Res. 150, 450-458. doi: 10.1016/j.schres.2013.09.016

Zhang, Y., Schuff, N., Jahng, G. H., Bayne, W., Mori, S., Schad, L., et al. (2007). Diffusion tensor imaging of cingulum fibers in mild cognitive impairment and Alzheimer disease. Neurology 68, 13-19. doi: 10.1212/01.wnl.0000250326. 77323.01

Conflict of Interest Statement: The authors declare that the research was conducted in the absence of any commercial or financial relationships that could be construed as a potential conflict of interest.

Received: 14 August 2013; accepted: 23 January 2014; published online: 11 February 2014.

Citation: Steinmann S, Leicht $G$ and Mulert C (2014) Interhemispheric auditory connectivity: structure and function related to auditory verbal hallucinations. Front. Hum. Neurosci. 8:55. doi: 10.3389/fnhum.2014.00055

This article was submitted to the journal Frontiers in Human Neuroscience.

Copyright $\odot 2014$ Steinmann, Leicht and Mulert. This is an open-access article distributed under the terms of the Creative Commons Attribution License (CC BY). The use, distribution or reproduction in other forums is permitted, provided the original author(s) or licensor are credited and that the original publication in this journal is cited, in accordance with accepted academic practice. No use, distribution or reproduction is permitted which does not comply with these terms. 